\title{
Atorvastatin in the treatment of Lithium- induced nephrogenic diabetes insipidus: the protocol of a randomized controlled trial
}

Jocelyn Fotso Soh ${ }^{1 *}$ DD, Susana G. Torres-Platas ${ }^{1}$, Serge Beaulieu², Outi Mantere ${ }^{2}$, Robert Platt ${ }^{3}$, Istvan Mucsi ${ }^{4}$, Sybille Saury ${ }^{2}$, Suzane Renaud ${ }^{2}$, Andrea Levinson ${ }^{5}$, Ana C. Andreazza ${ }^{5}$, Benoit H. Mulsant ${ }^{5}$, Daniel Müller, Ayal Schaffer ${ }^{6}$, Annemiek Dols ${ }^{7}$, Pablo Cervantes ${ }^{8}$, Nancy CP Low ${ }^{8}$, Nathan Herrmann ${ }^{6}$, Birgitte M. Christensen ${ }^{9}$, Francesco Trepiccione ${ }^{10}$, Tarek Rajji ${ }^{5}$ and Soham Rej ${ }^{1,2}$

\begin{abstract}
Background: Lithium is the gold-standard treatment for bipolar disorder, is highly effective in treating major depressive disorder, and has anti-suicidal properties. However, clinicians are increasingly avoiding lithium largely due to fears of renal toxicity. Nephrogenic Diabetes Insipidus (NDI) occurs in 15-20\% of lithium users and predicts a 2-3 times increased risk of chronic kidney disease (CKD). We recently found that use of statins is associated with lower NDI risk in a cross-sectional study. In this current paper, we describe the methodology of a randomized controlled trial $(\mathrm{RCT})$ to treat lithium-induced NDI using atorvastatin.

Methods: We will conduct a 12-week, double-blind placebo-controlled RCT of atorvastatin for lithium-induced NDI at McGill University, Montreal, Canada. We will recruit 60 current lithium users, aged 18-85, who have indicators of $\mathrm{NDI}$, which we defined as urine osmolality $(\mathrm{UOsm})<600 \mathrm{mOsm} / \mathrm{kg}$ after $10-\mathrm{h}$ fluid restriction. We will randomize patients to atorvastatin ( $20 \mathrm{mg} /$ day) or placebo for 12 weeks. We will examine whether this improves measures of NDI: UOsm and aquaporin (AQP2) excretion at 12-week follow-up, adjusted for baseline.
\end{abstract}

Results: Not applicable.

Conclusion: The aim of this clinical trial is to provide preliminary data about the efficacy of atorvastatin in treating NDI. If successful, lithium could theoretically be used more safely in patients with a reduced subsequent risk of CKD, hypernatremia, and acute kidney injury (AKI). If future definitive trials confirm this, this could potentially allow more patients to benefit from lithium, while minimizing renal risk.

Trial registration: ClinicalTrials.gov NCT02967653. Registered in February 2017.

Keywords: Lithium, Nephrogenic diabetes insipidus, Kidney function, Atorvastatin, Placebo, Urinary osmolality, Randomized clinical trial

\footnotetext{
* Correspondence: jfs702@mun.ca

${ }^{1}$ Geri-PARTy Research Group, Jewish General Hospital/Lady Davis Institute,

McGill University, 4333 Cote Ste-Catherine, Montreal, QC, H3T, 1E4, Montreal,

Canada

Full list of author information is available at the end of the article
}

(c) The Author(s). 2018 Open Access This article is distributed under the terms of the Creative Commons Attribution 4.0 International License (http://creativecommons.org/licenses/by/4.0/), which permits unrestricted use, distribution, and reproduction in any medium, provided you give appropriate credit to the original author(s) and the source, provide a link to the Creative Commons license, and indicate if changes were made. The Creative Commons Public Domain Dedication waiver (http://creativecommons.org/publicdomain/zero/1.0/) applies to the data made available in this article, unless otherwise stated. 


\section{Background}

Lithium remains the gold standard treatment for bipolar disorder and is associated with a better treatment response in $30-40 \%$ of patients compared to other bipolar pharmacotherapies [1,2]. Lithium is also effective in treatment-resistant depression [1], has been associated with reduced suicidality [3], and is even being investigated in a number of neurological conditions such as dementia and stroke $[4,5]$. Lithium is valuable considering the difficulty in achieving and maintaining symptomatic remission in the majority of patients with mood disorders [6]. Furthermore, lithium responders often do not respond well to alternative pharmacotherapies [5]. Rates of disability in people with mood disorders contribute to significant personal, family and societal costs: estimates of these total costs in the U.S. range from \$US30-40 billion $[7,8]$.

Despite the effectiveness of lithium, many clinicians avoid using it, with prescribing rates declining markedly in the past 2 decades, particularly in America where only $8 \%$ of bipolar disorder patients receive lithium [9]. This is likely in large part due to the perception of serious potential adverse effects associated with lithium use compared to other treatment options [10].

Of particular clinical concern has been lithium's potentially irreversible effects on kidney function: in addition to acute kidney injury (AKI), lithium has also been associated with a 2 times increased risk of chronic kidney disease (CKD) [11-13]. CKD, in turn, is associated with increased mortality, physical health co-morbities, decreased quality of life, family/caregiver burden, and major health care costs [14].

The exact mechanism whereby lithium causes CKD is not known. The most prominent pathology seen in patients with CKD attributed to lithium is interstitial nephropathy [15]. This points to a potential link between lithium related NDI and CKD. NDI is characterized by excessive production of dilute urine $(>3 \mathrm{~L} / 24 \mathrm{~h})$ due to tubular resistance to antidiuretic hormone [16]. Half of lithium users have difficulty concentrating urine: e.g. urine osmolality (UOsm) $<600 \mathrm{mOsm} / \mathrm{kg}$ following overnight water restriction [15]. Lithium-induced NDI is problematic: in addition to being related to frequent AKI and hypernatremia [17], NDI is also associated with a 2-3 times increased risk of chronic kidney disease (CKD) $[12,17]$. Thus, treating lithium-induced NDI could potentially help prevent CKD.

Presently, amiloride is the only medication with evidence from small randomized controlled trials (RCTs) $(n=9, n=11)$ to treat NDI, with a handful of other diuretics and non-steroidal anti-inflammatory agents (NSAIDs) having even lower-quality evidence $[15,18]$. However, these drugs have been associated with acute lithium-level elevations of up to $33-50 \%$, which could increase the risk of acute central nervous system and renal toxicity $[19,20]$. Therefore, there is a need for novel, well-tolerated treatments for lithium-induced NDI.

In a recent cross-sectional study, we found that statins were associated with a lower risk of NDI amongst lithium users $(n=71)$. In this study, lithium users who were also using statins were at lower risk $(0 \% ; 0 / 17)$ of developing NDI compared to lithium users who were not on statins $(20.4 \% ; 11 / 54)$ [21]. Two mice studies also demonstrated the effectiveness of statins in genetic forms of NDI, where statins increased the expression of membrane aquaporin 2 in renal tubules and doubled the urine osmolality $[22,23]$. Atorvastatin and other statins are well-tolerated and have minimal adverse effects on cognition and mood [24], but have positive effects on cardiovascular risk prevention [9], making it a palatable medication intervention for patients with mental disorders using lithium. In this present paper, we describe the first RCT to evaluate the effectiveness of atorvastatin for treating NDI in a population of lithium users.

\section{Methods \\ Study design and study sample}

This is a double-blind, placebo-controlled RCT examining the effectiveness of atorvastatin versus placebo in the treatment of lithium-induced NDI. The study will take place at three tertiary care mental health sites in Montreal, Canada: Douglas Mental Health University Institute (DMHUI), Jewish General Hospital (JGH) and McGill University Health Centre (MUHC).

The study will involve treatment with atorvastatin (20 $\mathrm{mg} /$ day) or placebo over a period of 12 weeks. We are aiming to recruit sixty individuals between the ages of 18 and 85 with any psychiatric diagnosis who are currently taking lithium for at least 2 months and have a urine osmolality $<600 \mathrm{mOsm} / \mathrm{kg}$ after 10-h water restriction. We opted for a urinary osmolality of $<600 \mathrm{mOsm} / \mathrm{Kg}$ : patients with $<600 \mathrm{mOsm} / \mathrm{kg}$ are at risk of future NDI-related complications, such as CKD and they constitute a large percentage of lithium users (50\% of patients). Other studies have used similar, if not less conservative definitions for NDI [25], which are nonetheless of clinical importance and utility [15]. Other authors have used similar or more liberal definitions in NDI treatment studies [25].

The full inclusion and exclusion criteria can be found in Table 1.

\section{Recruitment procedures}

This study has been approved by the local institutional review board (IRBs) of each participating center. Participants are introduced to the study by their treating physician. Interested participants meet with the study 
Table 1 Inclusion and exclusion criteria

\begin{tabular}{ll}
\hline Inclusion Criteria & Exclusion Criteria \\
\hline $\begin{array}{l}\text { Between ages 18-85 } \\
\text { Bipolar Diagnosis in any phase of illness: euthymic, depressed } \\
\text { or hypomanic. Any schizoaffective diagnosis. }\end{array}$ & $\begin{array}{l}\text { Already taking statins (at least } 6 \text { weeks prior to screening) } \\
\text { History of adverse reaction to statins; allergy to statins }\end{array}$ \\
$\begin{array}{l}\text { Diagnosed with NDI, defined by routine } 10 \mathrm{~h} \text { water restriction } \\
\text { urine osmolality }<600 \mathrm{mOsm} / \mathrm{Kg}\end{array}$ & $\begin{array}{l}\text { Contraindications to statin use: pregnancy or lactation, use of } \\
\text { fibrates and heavy alcohol use }\end{array}$ \\
$\begin{array}{ll}\text { Currently taking lithium medication (at least } 2 \text { months) } \\
\text { Able and willing to give consent or deemed to have moderate-severe cognitive }\end{array}$ \\
& $\begin{array}{l}\text { disturbances by treating clinician } \\
\text { Activer disease }\end{array}$ \\
\hline
\end{tabular}

research assistant for more details and written informed consent. Patients are then screened for NDI $(<600 \mathrm{mOsm} / \mathrm{Kg}$ after $10 \mathrm{~h}$ of water-restriction) by their treating clinicians as part of routine clinic visits or by the research assistant in a subsequent visit. Participant flow through the study is described in Fig. 1a and b.

\section{Overview of study procedures}

In this double-blind RCT, sixty patients will receive 12 weeks of either treatment $(20 \mathrm{mg} /$ day atorvastatin) or placebo. The study will require three visits at baseline, 4-week and 12-week follow-up, for which participants will be compensated. During each visit, blood and urine samples after $10 \mathrm{~h}$ of water restriction and $12 \mathrm{~h}$ of fasting will be requested. In addition to the primary and secondary outcomes, patients will be monitored for safety using blood tests for liver function (AST and ALT), cholesterol (e.g. low-density lipoprotein (LDL)) and lithium toxicity (lithium levels, estimated glomerular filtration rate (eGFR)). Patients will also complete questionnaires about their medical history and mental health. Mood assessments for depression and mania symptoms will be completed at baseline, 4-week and 12-week follow-up, including the Montgomery-Asberg Depression Rating Scale (MADRS) [26], Young Mania Rating Scale (YMRS) [27], and the Quick Inventory of Depressive Symptoms, Self-report-16 (QIDS-16) [28], Altman Self-Rating Mania Scale (ASMRS) [29].

The primary outcome is change in $10 \mathrm{~h}$ water restriction urine osmolality $(\mathrm{mOsm} / \mathrm{kg})$ and will be assessed at baseline, 4-week and 12-week follow-up visits (Table 2).

\section{Randomization and concealment}

After eligibility is confirmed and patients have given consent, patients will be randomized in a $1: 1$ ratio to receive either $20 \mathrm{mg}$ per day of atorvastatin (treatment group) or placebo (control group). We are using a stratified randomization, with two strata: 1. Diagnosis (bipolar disorder, other diagnosis (e.g. depression, schizoaffective disorder), and 2. McGill hospital site (Douglas Mental Health Institute, Jewish General Hospital (JGH), and
McGill University Health Centre (MUHC)). An independent entity, the Lady Davis Institute Data Management Unit is responsible for setting up data management software (Redcap) and randomization. Allocation of participants to groups is concealed in an envelope that only the pharmacy at the Douglas Institute has access to. The patients, clinicians, researcher and outcome assessors will all be blind to the treatment allocation. An independent statistician will perform the statistical analyses.

\section{Assessments \\ Outcomes measures}

Our primary outcome will be urinary osmolality after 10 -h water restriction. This short-period water restriction was selected given that longer periods of water restriction are often poorly tolerated by patients, especially those on lithium treatment [30]. Clinically, the 10-h water restriction assessment of urine osmolality is often used and is highly specific and sensitive for NDI $[25,31,32]$. We will assess the raw urine osmolality value at 12-weeks, adjusted for baseline. An increase in urine osmolality $(\mathrm{mOsm} / \mathrm{Kg}$ ) values adjusted for baseline will be indicative of improvements in the kidney's ability to concentrate urine [33, 34]. We hypothesize that higher osmolality values will be observed compared to baseline in patients randomized to atorvastatin.

We chose measures of aquaporin 2 excretion and self-reports of daily fluid intake as the secondary outcomes. Aquaporins are channels involved in water reabsorption in the collecting ducts of the kidney [35]. Assessment of aquaporin excretion in urine will be indicative of the expression of the aquaporin channels and water reabsorption capabilities of the kidneys, allowing further confirmation of whether: 1) NDI pathology is present and 2) whether it is being modified by atorvastatin treatment. Furthermore, changes in daily fluid intake have been highly correlated with both urinary osmolality and the gold-stand as a characteristic of NDI $[30,31,36]$. Additionally, we supplement these secondary measures with self-reported frequency of nighttime and daytime polyuria. 


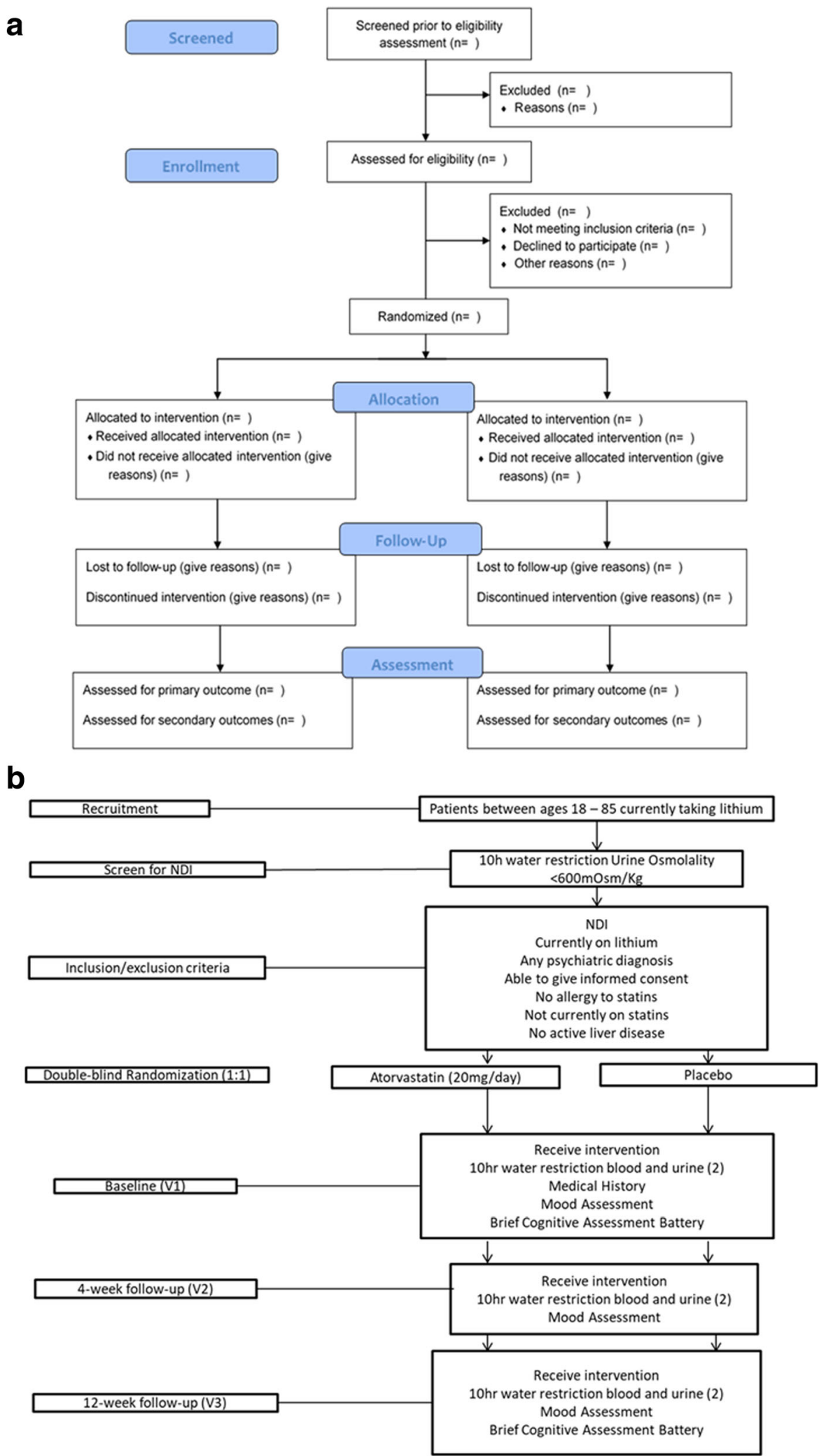

Fig. 1 a Flow chart - Flow of Study Participants through the RCT. b Consort diagram for reporting randomized controlled trials (Eldridge et al., 2016 [40]) 
Table 2 Baseline and follow-up visits schedule of events and assessments

\begin{tabular}{lllll}
\hline Assessment & Screen & Baseline & 4-week & 12-week \\
\hline Blood & $\sqrt{ }$ & $\sqrt{ }$ & $\sqrt{ }$ & $\sqrt{ }$ \\
Urine osmolality & $\sqrt{ }$ & $\sqrt{ }$ & $\sqrt{ }$ & $\sqrt{ }$ \\
Medical History & $\sqrt{ }$ & $\sqrt{ }$ & & $\sqrt{ }$ \\
Mood Assessment & & $\sqrt{ }$ & $\sqrt{ }$ & $\sqrt{ }$ \\
1.MADRS & $\sqrt{ }$ & $\sqrt{ }$ & $\sqrt{ }$ \\
2.YMRS & $\sqrt{ }$ & $\sqrt{ }$ & $\sqrt{ }$ \\
3. QIDS & $\sqrt{ }$ & $\sqrt{ }$ & $\sqrt{ }$ \\
4. Altman self-rating mania scale & & $\sqrt{ }$ & $\sqrt{ }$ & $\sqrt{ }$ \\
\hline
\end{tabular}

\section{Safety and tolerability measures}

Safety and tolerability outcome measures will be assessed including blood tests at routine and follow-up visits to ensure patient health (monitoring atorvastatin-related adverse effects). Blood tests will allow the monitoring of serum lithium levels, lipid profile (including LDL levels), creatinine kinase, liver function enzymes, calcium, thyroid stimulating hormone, and electrolytes. Patient reported symptoms - e.g. for myalgias - are also reported. In addition, mood assessments - YMRS, MADRS, QIDS, and AMSRS assessments during follow-up visits will be used to monitor mood changes in patients. YMRS is an eleven-item scale to assess manic symptoms in individuals [27]. MADRS is a ten-item scale for a measure of depressive symptoms [26]. QIDS is a twenty-item self-report measure on depression [28], whereas the ASRMS is a five-item self-report measure for manic symptoms [29].

\section{Baseline demographic and clinical characteristics}

We collect information on a number of baseline demographic and clinical characteristics, including: age, sex, body mass index (BMI), psychiatric and other medication history including duration of lithium use and dosage. Of these factors, age, lithium-level, duration, dosage and anti-psychotic use have been associated with NDI [17, 30].

\section{Planned analysis}

We will use independent two-tailed Student's t-test (or non-parametric equivalent) to compare the primary outcome (raw score values of urinary osmolality at 12-weeks, adjusted for baseline) and secondary outcomes (self-reported fluid intake and variation in aquaporin 2 protein abundance in urinary exosomes at 12-week follow-up, adjusted for baseline) between the treatment (atorvastatin) and control (placebo) groups. The consensus in the statistics RCT literature is that clinical trial outcomes should not be "change from baseline", but rather the raw score of an outcome, adjusted for the baseline value [37]. Randomization is expected to balance potential confounders between the treatment and placebo groups since both groups are anticipated to have roughly equal baseline characteristics, which we will assess using t-tests (or non-parametric equivalent) and chi-squared tests, as appropriate. However, in the event that any important baseline characteristic differs between groups, we will use multiple linear regression to control for that particular characteristic.

\section{Sample size justification}

On repeated measures ANOVA, 60 study completers will allow us to observe an effect size of 0.34 at two-tailed alpha $=0.05$ and Power $(1$-Beta $)=0.8$ [38]. In our previous cross-sectional findings in chronic lithium users, the effect size of statins on NDI was 0.33 [21], however that was not an intervention study. We acknowledge that there is relatively little data at the current time to justify the sample size. This RCT will provide the necessary and adequate data to plan a subsequent larger RCT [39].

\section{Discussion}

This paper described the methodology of a clinical trial to assess the effectiveness of atorvastatin in treating NDI in lithium users. If atorvastatin is ultimately found to be effective for NDI, some of the risk associated to CKD, hypernatremia and AKI attributable to lithium could be attenuated, allowing a greater number of persons to use lithium more safely. Minimizing these risks of kidney disease associated with lithium use is important because it could potentially prevent acute and chronic medical health care services related to renal disease and psychiatric illness, possibly saving significant personal and health care costs worldwide.

The present study is the first RCT of atorvastatin in the treatment of Li-induced NDI. This study represents an advance in methodological rigor compared to previous studies examining the treatment of NDI [17]. These studies were similar in design to the present study, however these used very small sample sizes $(n=9, n=11)[18,25]$. Hydrochlorothiazide (HCTZ), other diuretics, non-steroidal anti-inflammatory drugs (NSAIDs), and aspirin (ASA) have also been tried in NDI, but the studies supporting their use are almost exclusively from small uncontrolled trials/chart reviews [17]. In addition, there are limitations to the use of amiloride, diuretics, NSAIDs and other medications previously tested to treat NDI: these medications can lead to elevations in lithium levels contributing to increased risk of lithium-associated CNS and acute renal toxicity. We present an RCT with comparatively large sample size $(n=60)$, which will provide preliminary data to guide larger-scale international collaborative study.

\section{Limitations}

There are some limitations to this proposed study. For our primary outcome, urine osmolality, we used a threshold of $<600 \mathrm{mOsm} / \mathrm{Kg}$ after $10 \mathrm{~h}$ of water 
restriction to define NDI and allow patients to enter the study. NDI is ideally more conservatively measured with an even lower urine osmolality threshold that is more specific/sensitive for NDI. However, a urine osmolality $<600 \mathrm{mOsm} / \mathrm{Kg}$ is a clinically prevalent entity $(50 \%$ of patients), associated with poor renal outcomes and is nonetheless of clinical importance and utility [15]. Other trials have used similar thresholds (e.g. < $700 \mathrm{mOsm} / \mathrm{kg}$ ) under similar 8-10-h water restriction periods, has been termed as "partial NDI" [25]. The overnight water restriction urine osmolality has relatively high sensitivity/specificity for NDI compared to the gold-stander 24-h urine collection, particularly in combination with self-reported fluid intake, which we are also measuring [31]. In addition, we plan to complete a sub-group analysis of patients with a urine osmolality $<300 \mathrm{mOsm} / \mathrm{Kg}$.

\section{Conclusion}

To our knowledge, this is the first planned RCT for the treatment of NDI using atorvastatin. Future trials could 1) further assess the effectiveness of atorvastatin in treating lithium-induced NDI in a larger sample and 2) examine atorvastatin in the prevention of lithium-induced NDI and CKD. If found effective, atorvastatin could ultimately be combined with lithium regimens to treat/prevent NDI, which in turn could reduce the risk of CKD and other renal adverse events in lithium users. This could potentially allow clinicians to prescribe lithium to a larger number of patients with bipolar disorder, depression and other psychiatric/neurological disorders: Both maximizing positive potential therapeutic benefits of lithium, while minimizing renal adverse events.

\section{Abbreviations \\ AKI: Acute Kidney Injury; ALT: Alanine Aminotransferase Test; ANOVA: Analysis of Variance; AQP2: Aquaporin 2; ASA: Aspirin; ASRMS: Altman Self-Rating Mania Scale; AST: Aspartate Aminotransferase Test; BMI: Body Mass Index; CKD: Chronic Kidney Disease; eGFR: Estimated Glumerular Filtration Rate; HCTZ: Hydrochlorothiazide; IRB: Institutional Review Board; LDL: Low Density Lipoprotein; MADRS: Montgomery Asberg Depression Rating Scale; NDI: Nephrogenic Diabetes Insipidus; NSAIDs: Non-Steriodal Anti-Inflammatory Drugs; QIDS: Quick Inventory of Depressive Symptoms; RCT: Randomized Controlled Trial; SCIP: Screen for Cognitive Impairment in Psychiatry; UOsm: Urinary Osmolality; YMRS: Young Mania Rating Scale}

\section{Ethics approval and consent to participants}

This study has been approved by the institutional review board (IRBs) at the Douglas Mental Health University Institute, McGill University Health Centre and Jewish General Hospital (Montreal, Canada), and in this ongoing randomized controlled trial, all particpants will provide informed written consent.

\section{Funding}

This study is supported by a biomedical research grant from the Kidney Foundation of Canada (KFoC). Grant number: KFOC170016.

This funding would aid in the recruitment and data collection, analysis and interpretation of the data, and the writing of manuscripts for publication in scientific journals. Dr. Rej has received salary support from the Fonds de Recherche Santé Québec (FRQS) Chercheur Boursier Clinicien Junior 1 Award.

\section{Authors' contributions}

JFS was involved in study design and conception, ongoing data collection and played a leading role in manuscript preparation. SGT, SB, SR, OM and SS made substantial contribution to the conception and design of the study. RP has contributed in the study design and played a leading role in the methodology for statistical analysis. IM, AL, AA, BM, BMC, FT, DM, AS, AD, TR and $\mathrm{NH}$ have contributed to the design and methodology and played a leading role in planned analyses. PC and NL have contributed to the design and methodology of the study. SR played a leading role in the conception and design of the study and has contributed significantly in the revision of the manuscript. All authors revised manuscript for important intellectual content, gave final approval of the version to be published and agree to be accountable for all aspects of the work.

\section{Consent for publication}

We consent to publishing this protocol paper.

\section{Competing interests}

Dr. Rej has an investigator-initiated research grant from Satellite Healthcare (a Dialysis Company).

\section{Publisher's Note}

Springer Nature remains neutral with regard to jurisdictional claims in published maps and institutional affiliations.

\section{Author details}

${ }^{1}$ Geri-PARTy Research Group, Jewish General Hospital/Lady Davis Institute, McGill University, 4333 Cote Ste-Catherine, Montreal, QC, H3T, 1E4, Montreal, Canada. ${ }^{2}$ Douglas Mental Health University Institute and Department of Psychiatry, McGill University, Montreal, Canada. ${ }^{3}$ Department of Epidemiology, Biostatistics and Occupational Health, McGill University Health Centre, Montreal, Canada. ${ }^{4}$ Division of Nephrology, University Health Network, University of Toronto (UofT), Toronto, Canada. ${ }^{5}$ Department of Psychiatry, Centre for Addiction and Mental Health \& Department of Psychiatry, University of Toronto, Toronto, Canada. ${ }^{6}$ Department of Psychiatry, Sunnybrook Research Institute, University of Toronto, Toronto, Canada. ${ }^{7}$ Department of Psychiatry, GGZ, Geest, Amsterdam, the Netherlands.

${ }^{8}$ Department of Psychiatry, McGill University Health Centre, Montreal, Canada. ${ }^{9}$ Department of Biomedicine, University of Aarhus, Aarhus, Denmark.

${ }^{10}$ Division of Nephrology, University of Naples, Naples, Italy.

Received: 21 April 2018 Accepted: 14 June 2018

Published online: 16 July 2018

\section{References}

1. Yatham LN, Kennedy SH, Parikh SV, Schaffer A, Beaulieu S, Alda M, O'Donovan C, Macqueen G, McIntyre RS, Sharma V, et al. Canadian network for mood and anxiety treatments (CANMAT) and International Society for Bipolar Disorders (ISBD) collaborative update of CANMAT guidelines for the management of patients with bipolar disorder: update 2013. Bipolar Disord. 2013;15(1):1-44.

2. Alda M. Lithium in the treatment of bipolar disorder: pharmacology and pharmacogenetics. Mol Psychiatry. 2015;20(6):661-70.

3. Oquendo MA, Galfalvy HC, Currier D, Grunebaum MF, Sher L, Sullivan GM, Burke AK, Harkavy-Friedman J, Sublette ME, Parsey RV, et al. Treatment of suicide attempters with bipolar disorder: a randomized clinical trial comparing lithium and valproate in the prevention of suicidal behavior. Am J Psychiatry. 2011;168(10):1050-6.

4. Gerhard T, Devanand DP, Huang C, Crystal S, Olfson M. Lithium treatment and risk for dementia in adults with bipolar disorder: population-based cohort study. Br J Psychiatry. 2015;207(1):46-51.

5. Rybakowski JK. Response to lithium in bipolar disorder: clinical and genetic findings. ACS Chem Neurosci. 2014;5(6):413-21.

6. El-Mallakh RS, Vohringer PA, Ostacher MM, Baldassano CF, Holtzman NS, Whitham EA, Thommi SB, Goodwin FK, Ghaemi SN. Antidepressants worsen rapid-cycling course in bipolar depression: a STEP-BD randomized clinical trial. J Affect Disord. 2015;184:318-21.

7. Kleinman L, Lowin A, Flood E, Gandhi G, Edgell E, Revicki D. Costs of bipolar disorder. Pharmacoeconomics. 2003;21(9):601-22.

8. Murray CJ, Atkinson C, Bhalla K, Birbeck G, Burstein R, Chou D, Dellavalle R, Danaei G, Ezzati M, Fahimi A, et al. The state of US health, 1990-2010: burden of diseases, injuries, and risk factors. JAMA. 2013;310(6):591-608. 
9. Deedwania PC. Statins in chronic kidney disease: cardiovascular risk and kidney function. Postgrad Med. 2014;126(1):29-36.

10. Baldessarini R, Henk H, Sklar A, Chang J, Leahy L. Psychotropic medications for patients with bipolar disorder in the United States: polytherapy and adherence. Psychiatr Serv. 2008;59(10):1175-83.

11. Rej S, Herrmann N, Shulman K, Fischer HD, Fung K, Harel Z, Gruneir A. Lithium use, but not valproate use, is associated with a higher risk of chronic kidney disease in older adults with mental illness. J Clin Psychiatry. 2017;78(8):e980-5.

12. Rej S, Shulman K, Herrmann N, Harel Z, Fischer HD, Fung K, Gruneir A. Prevalence and correlates of renal disease in older lithium users: a population-based study. Am J Geriatr Psychiatry. 2014;22(11):1075-82.

13. Shine $B$, McKnight $R$, Leaver $L$, Geddes J. Long-term effects of lithium on renal function-Authors' reply. Lancet. 2015;386(10007):1944.

14. Lilly SM, Mortensen EM, Frei CR, Pugh MJ, Mansi IA. Comparison of the risk of psychological and cognitive disorders between persistent and nonpersistent statin users. Am J Cardiol. 2014;114(7):1035-9.

15. Boton R, Gaviria M, Batlle DC. Prevalence, pathogenesis, and treatment of renal dysfunction associated with chronic lithium therapy. Am J Kidney Dis. 1987;10(5):329-45

16. Grunfeld JP, Rossier BC. Lithium nephrotoxicity revisited. Nat Rev Nephrol. 2009;5(5):270-6.

17. Rej S, Herrmann N, Shulman K. The effects of lithium on renal function in older adults-a systematic review. J Geriatr Psychiatry Neurol. 2012;25(1):51-61.

18. Batlle DC, von Riotte AB, Gaviria M, Grupp M. Amelioration of polyuria by amiloride in patients receiving long-term lithium therapy. N Engl J Med. 1985;312(7):408-14.

19. Juurlink DN, Mamdani MM, Kopp A, Rochon PA, Shulman Kl, Redelmeier DA Drug-induced lithium toxicity in the elderly: a population-based study. J Am Geriatr Soc. 2004;52(5):794-8.

20. Phelan KM, Mosholder AD, Lu S. Lithium interaction with the cyclooxygenase 2 inhibitors rofecoxib and celecoxib and other nonsteroidal anti-inflammatory drugs. J Clin Psychiatry. 2003;64(11):1328-34.

21. Elie D, Segal M, Low NC, Mucsi I, Holcroft C, Shulman K, Looper KJ, Rej S. Statins in the prevention of lithium-associated diabetes insipidus: preliminary findings. Kidney Int. 2015;87(4):862.

22. Li W, Zhang Y, Bouley R, Chen Y, Matsuzaki T, Nunes P, Hasler U, Brown D, Lu HA. Simvastatin enhances aquaporin-2 surface expression and urinary concentration in vasopressin-deficient Brattleboro rats through modulation of rho GTPase. Am J Physiol Renal Physiol. 2011;301(2):F309-18.

23. Procino G, Milano S, Carmosino M, Barbieri C, Nicoletti MC, Li JH, Wess J, Svelto M. Combination of secretin and fluvastatin ameliorates the polyuria associated with X-linked nephrogenic diabetes insipidus in mice. Kidney Int. 2014;86(1):127-38.

24. Swiger KJ, Manalac RJ, Blumenthal RS, Blaha MJ, Martin SS. Statins and cognition: a systematic review and meta-analysis of short- and long-term cognitive effects. Mayo Clin Proc. 2013;88(11):1213-21.

25. Bedford JJ, Weggery S, Ellis G, McDonald FJ, Joyce PR, Leader JP, Walker RJ. Lithium-induced nephrogenic diabetes insipidus: renal effects of amiloride. Clin J Am Soc Nephrol. 2008;3(5):1324-31.

26. Montgomery SA, Asberg M. A new depression scale designed to be sensitive to change. Br J Psychiatry. 1979;134:382-9.

27. Young RC, Biggs JT, Ziegler VE, Meyer DA. A rating scale for mania: reliability, validity and sensitivity. Br J Psychiatry. 1978;133:429-35.

28. Rush AJ, Trivedi MH, Ibrahim HM, Carmody TJ, Arnow B, Klein DN, Markowitz JC, Ninan PT, Kornstein S, Manber R, et al. The 16-item quick inventory of depressive symptomatology (QIDS), clinician rating (QIDS-C), and self-report (QIDS-SR): a psychometric evaluation in patients with chronic major depression. Biol Psychiatry. 2003;54(5):573-83.

29. Altman EG, Hedeker D, Peterson JL, Davis JM. The Altman self-rating mania scale. Biol Psychiatry. 1997;42(10):948-55.

30. Rej S, Segal M, Low NC, Mucsi I, Holcroft C, Shulman K, Looper K. The McGill geriatric Lithium-induced diabetes insipidus clinical study (McGLIDICS). Can J Psychiatr. 2014;59(6):327-34

31. Kinahan JC, NiChorcorain A, Cunningham S, Freyne A, Cooney C, Barry S, Kelly BD. Risk factors for polyuria in a cross-section of community psychiatric lithium-treated patients. Bipolar Disord. 2015;17(1):50-62.

32. van Melick EJ, Meinders AE, Hoffman TO, Egberts TC. Renal effects of longterm lithium therapy in the elderly: a cross-sectional study. Int J Geriatr Psychiatry. 2008;23(7):685-92.
33. Perrier E, Rondeau P, Poupin M, Le Bellego L, Armstrong LE, Lang F, Stookey J, Tack I, Vergne S, Klein A. Relation between urinary hydration biomarkers and total fluid intake in healthy adults. Eur J Clin Nutr. 2013;67(9):939-43.

34. Strippoli GF, Craig JC, Rochtchina E, Flood VM, Wang JJ, Mitchell P. Fluid and nutrient intake and risk of chronic kidney disease. Nephrology (Carlton). 2011;16(3):326-34.

35. Kamsteeg EJ, Heijnen I, van Os CH, Deen PM. The subcellular localization of an aquaporin-2 tetramer depends on the stoichiometry of phosphorylated and nonphosphorylated monomers. J Cell Biol. 2000;151(4):919-30.

36. Timmer RT, Sands JM. Lithium intoxication. J Am Soc Nephrol. 1999;10(3):666-74

37. Harrell FaS, J: biostatistics for biomedical research. In., 27/02/2018 edn; 2018.

38. Faul F, Erdfelder E, Buchner A, Lang A: G* Power Version 3.1. 7 [computer software]. Uiversität Kiel, Germany. In.; 2013.

39. Birkett MA, Day SJ. Internal pilot studies for estimating sample size. Stat Med. 1994;13(23-24):2455-63.

40. Eldridge SM, Chan CL, Campbell MJ, Bond CM, Hopewell S, Thabane L, Lancaster GA, group pc. CONSORT 2010 statement: extension to randomised pilot and feasibility trials. BMJ. 2016;355:15239.

\section{Ready to submit your research? Choose BMC and benefit from:}

- fast, convenient online submission

- thorough peer review by experienced researchers in your field

- rapid publication on acceptance

- support for research data, including large and complex data types

- gold Open Access which fosters wider collaboration and increased citations

- maximum visibility for your research: over $100 \mathrm{M}$ website views per year

At $B M C$, research is always in progress.

Learn more biomedcentral.com/submissions 